\title{
A Toolbox to Study Tissue Mechanics In Vivo and Ex Vivo
}

\author{
Sofia Moreira, Jaime A. Espina, Joana E. Saraiva, and Elias H. Barriga
}

\begin{abstract}
During vertebrate embryogenesis, tissues interact and influence each other's development to shape an embryo. While communication by molecular components has been extensively explored, the role of mechanical interaction between tissues during embryogenesis is just starting to be revealed. Addressing mechanical involvement in morphogenesis has traditionally been challenging mainly due to the lack of proper tools to measure and modify mechanical environments of cells in vivo. We have recently used atomic force microscopy (AFM) to show that the migration of the Xenopus laevis cephalic neural crest cells is triggered by stiffening of the mesoderm, a tissue that neural crest cells use as a migratory substrate in vivo. Interestingly we showed that the activity of the planar cell polarity (PCP) pathway is required to mediate this novel mechanical interaction between two tissues. In this chapter, we share the toolbox that we developed to study the role of PCP signaling in mesoderm cell accumulation and stiffening (in vivo) as well as the impact of mesoderm stiffness in promoting neural crest cell polarity and migration (ex vivo). We believe that these tools can be of general use for investigators interested in addressing the role of mechanical inputs in vivo and ex vivo.
\end{abstract}

Key words Collective cell migration (CCM), Planar cell polarity (PCP), Hydrogels, Atomic force microscopy (AFM), Neural crest (NC) cells, Mesoderm, Xenopus laevis

\section{Introduction}

During vertebrate embryonic development, morphogenetic processes shape a multilayered three-dimensioned embryo consisting of tissues derived from ectoderm, endoderm, and mesoderm [1]. Cells from these three germ layers functionally influence each other to generate differentiated tissues that organize in stereotypical ways to produce embryo forms [2]. The development of the nervous system is a typical illustration of the importance of this tissue interaction, whereby the brain and the spinal cord arise from dorsal ectoderm via induction by molecular signals derived from the underlying dorsal mesoderm (known by amphibian embryologists as the "Spemann's organizer") [3]. Another example is the

Sofia Moreira, Jaime A. Espina and Joana E. Saraiva contributed equally with all other contributors. 
development of the vertebrate eye, where interactions during gastrulation are required to give the prospective head ectoderm a lensforming bias [4]. Notably, the neural crest cells, a multipotent embryonic cell population, arise at the border of the neural plate owing to concerted actions of signaling pathways from neural, non-neural ectoderm, and their underlying mesoderm [5]. Thus, there is substantial evidence that tissues communicate through signaling molecules during vertebrate embryogenesis. However, until very recently, little was known about whether and how cells from different layers interact mechanically to influence each other. This aspect of the embryonic development was lagging behind. However, in the last decade, several advances have been made toward the generation of a mechanical framework of embryogenesis $[6,7]$. Most of these advances are made due to the development of reliable tools and the establishment of robust animal systems where mechanical cues can be measured and modified [8]. For instance, we recently demonstrated that migration of the Xenopus laevis cephalic neural crest cells (NCs) is mechanically triggered by stiffening of the mesoderm, the tissue that NCs use as a migratory substrate [6]. In this work we discovered that the source of this stiffening was an increase in mesodermal cell density owing convergent extension movements which are known to rely on the activity of the planar cell polarity pathway (PCP) [9]. These two processes-convergent extension of the mesoderm that starts early in gastrulation and NCs collective cell migration (CCM) - have been normally studied separately. Hence, our recent discoveries revealed that at least these two tissues can mechanically interact to coordinate morphogenesis by triggering the onset of CCM in vivo [6].

Demonstrating this point involved the use of a refined mechano-molecular toolbox aimed to determine the impact that modifying the activity of PCP in the mesoderm has on both mesoderm cell migration and stiffness, as well as on NCs polarity and migration. While several animal models are available to study tissue mechanics [10-12], we believe that Xenopus laevis is particularly appealing to study mechano-molecular feedback loops arising from mechanical interaction between tissues. Frog embryos are a robust system where mechanical manipulations can be easily applied and the impact of these manipulations on molecular and cellular outputs can be easily analyzed. Xenopus NCs also allow for in vivo and ex vivo analyses. This is particularly relevant when it comes to mechanical analyses as it allows to reproduce in vivo-like mechanical conditions in a controlled ex vivo environment [13]. Hence, in this article we share a detailed protocol that we hope will enable other investigators to study molecular details of mechano-molecular feedback loops in embryogenesis. Our work is organized in several sections, where we will explain how to specifically target dorsal mesoderm in order to molecularly modify PCP activity in this tissue (Subheading 3.1); how to determine the impact of PCP inhibition 
on mesoderm cell density (Subheading 3.2 ); and how to use atomic force microscopy (AFM) to determine the impact of PCP inhibition on mesoderm stiffness in vivo (Subheading 3.3). Here we will show for the first time our new setup that allows the use of AFM by researchers with minimal experience. Finally, we will introduce an ex vivo system where the impact of substrate stiffness on cell polarity can be easily analyzed (Subheading 3.4). In this system, the mesodermal stiffness values registered at non-migratory and migratory stages are mimicked into fibronectin-coated hydrogels. Altogether, we hope that our protocols can be adapted to other biological systems where mechano-molecular feedback loops also play a role in the context of not only cell migration but also in other biological processes.

\section{Materials}

\subsection{Microinjection}

\subsubsection{Embryo Medium}

\subsubsection{Plasmids} and $m R N A$
All the protocols described here are performed using different dilutions of a $1 \times$ stock solution of Marc's Modified Ringer's (MMR) media, which is described in detail below. All the media solutions need to be sterilized prior to use (autoclaved, filtered and/or using UV light).

1. Stock MMR solution $(\mathrm{l} \times)$ : to prepare $1 \mathrm{l}$, add $0.149 \mathrm{~g} \mathrm{KCl}$, $0.228 \mathrm{~g} \mathrm{MgSO}_{4} .6 \mathrm{H}_{2} \mathrm{O}, 0.294 \mathrm{~g} \mathrm{CaCl}_{2} .2 \mathrm{H}_{2} \mathrm{O}, 5.844 \mathrm{~g} \mathrm{NaCl}$, $0.0372 \mathrm{~g}$ EDTA, $1.191 \mathrm{~g}$ HEPES to $900 \mathrm{ml}$ distilled water. Adjust $\mathrm{pH}$ to 7.4 and complete the volume until 11 with sterile $\mathrm{ddH}_{2} \mathrm{O}$.

2. Embryo growth medium $(0.1 \times M M R)$ : to maintain embryo development, use the previously described $1 \times$ MMR stock solution and dilute it in sterile $\mathrm{ddH}_{2} \mathrm{O}$ to obtain a $0.1 \times$ MMR media.

3. Embryo dissection medium $(0.3 \times \mathrm{MMR})$ : to dissect neural crest cells, use the described $1 \times$ MMR stock solution and dilute in sterile $\mathrm{ddH}_{2} \mathrm{O}$ to obtain a $0.3 \times \mathrm{MMR}$ media.

4. Embryo injection medium $(0.3 \times$ MMR plus $4 \%$ Ficoll (PolySucrose 400)): for microinjections, prepare a $0.3 \times \mathrm{MMR}$ solution from the MMR stock solution using sterile $\mathrm{ddH}_{2} \mathrm{O}$. Add $4 \%$ Ficoll (PolySucrose 400), then filter and use for injection.

1. Plasmid template for Dsh-DEP ${ }^{+}$: The plasmid construct pCS2Dsh-DEP ${ }^{+}$, kindly provided by Dr. M Tada (UCL) [6], was used to produce the RNA encoding Dsh-DEP ${ }^{+}$. This is a dominant-negative form of disheveled whose microinjection has been widely used to inhibit PCP specifically in the meso$\operatorname{derm}[6,9,14]$. 


\subsubsection{Microinjection} Equipment

\subsection{4}

Immunofluorescence

\subsection{AFM \\ Measurements}

\subsection{Polyacrylamide Hydrogels}

2. Plasmid template for mGFP (membrane marker): The plasmid pCS2-mGFP [6] was used to produce the RNA encoding for a membrane targeted version of GFP.

3. mRNA transcription: prior transcription, $\mathrm{pCS} 2-\mathrm{Dsh}-\mathrm{DEP}^{+}$or pCS2-mGFP were linearized with NotI and purified with a standard DNA purification kit. Linearized plasmids were used as templates for in vitro synthesis of capped mRNA that was carried out with a "mMessage mMachine ${ }^{\text {TM }}$ SP6 Transcription Kit" (Invitrogen ${ }^{\mathrm{TM}}$ ), according to the manufacturer's instructions. Use always RNase-free conditions.

1. Microinjector: mRNA injections were performed using a PM 1000 Cell Microinjector (from MicroData Instrument, Inc.).

2. Micropipette puller: capillary needles for microinjections were prepared from glass capillary tubes using a micropipette puller (Narishige PC-100), according to manufacturer's instructions.

3. Fine forceps Dumont \#5.

4. Incubator: Peltier-cooled incubator IPP110eco (Memmert), with temperature between $13.5^{\circ} \mathrm{C}$ and $14.5^{\circ} \mathrm{C}$.

1. Fixing solution: $4 \%$ formaldehyde in PBS $(1 \times)$ with $0.3 \%$ Triton $\mathrm{X}-100$.

2. Blocking solution (BS): 10\% Normal Goat Serum (NGS) diluted in PBS $(1 \times)$.

3. Primary antibody solution: anti-fibronectin ( $\mathrm{Fn})(\mathrm{mAb} 4 \mathrm{H} 2$ anti-FN from DSHB) in BS.

4. Secondary antibody solution with DAPI: Alexa Fluor 555 (anti-mouse) and DAPI $(1000 \times)$ in BS.

5. Washing solutions: PBS $(1 \times)$ or PBS $(1 \times)$ with $0.1 \%$ Tween-20.

1. Plasticine dishes for dissection and holding the embryos.

2. AFM probes: while users can prepare their own AFM probes (e.g., gluing beads to tipless cantilevers), we strongly recommend acquisition of cantilevers containing pre-coated colloidal spheres with spring constants between 0.01 and $0.03 \mathrm{~N} / \mathrm{m}$ (soft cantilevers) (see Note $\mathbf{1}$ ). These are spherical probes, which when interacting with samples lead to deflection of the cantilever without penetrating soft material [15].

3. SpReference samples: Standard Spectroscopy Mode Kit (Nanosurf).

1. Glass hydrophilic coating solution: 14:1:1 (volume) ethanol (100\%):acetic acid:PlusONE bind-silane (17-1330-01 GE Health Care) to coat $76 \mathrm{~mm} \times 24 \mathrm{~mm}$ glass slides. 
2. Glass hydrophobic coating solution: PlusONE repel-silane (17-1332-01 GE Health Care) to coat 13-mm diameter $\times 0.1 \mathrm{~mm}$ glass coverslips (No. 1- Cat 631-0149 VWR International).

3. Polyacrylamide (PAA) soft hydrogel mix: $550 \mu$ hydrochloric acid $(\mathrm{HCl}) 7.6 \mathrm{mM}, 350 \mu$ d double-distilled water $\left(\mathrm{ddH}_{2} \mathrm{O}\right)$, $0.5 \mu \mathrm{l} \quad \mathrm{N}, \mathrm{N}, \mathrm{N}^{\prime}, \mathrm{N}^{\prime}$-tetramethylethylenediamine (TEMED) (Sigma), $20 \mu \mathrm{l}$ bis-acrylamide 2\% (BioRad), $70 \mu$ acrylamide $40 \%$ (BioRad) and $5 \mu \mathrm{l}$ fluorescent beads $0.2 \mu \mathrm{M}$ (Invitrogen), add $5 \mu$ ammonium persulfate (APS) $10 \%$ (GE Health Care) just before use.

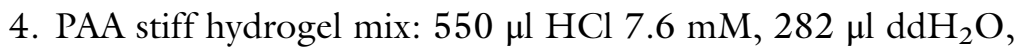
$0.5 \mu \mathrm{l}$ TEMED (Sigma), $25 \mu \mathrm{l}$ bis-acrylamide 2\% (BioRad), $137 \mu \mathrm{l}$ acrylamide $40 \%$ (BioRad) and $4 \mu$ fluorescent beads $0.2 \mu \mathrm{M}$ (Invitrogen), add $5 \mu \mathrm{l}$ APS 10\% (GE Health Care) just before use.

5. $10 \mathrm{mM}$ HEPES buffer (to wash the excess of reagents after polymerization).

6. Gel functionalization solution (see Note 2): Prepare a stock solution of $0.975 \mathrm{~g} 2$-Morpholinoethanesulfonic acid monohydrate (MES), and $0.57 \mathrm{~g} \mathrm{~N}$-hydroxysuccinimide (NHS)

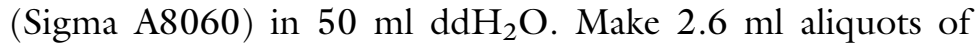
MES-NHS and store at $-20{ }^{\circ} \mathrm{C}$; Prepare $0.1 \mathrm{~g}$ aliquots of 1-ethyl-3-(3-dimethylaminopropyl) carbodiimide hydrochloride $(\mathrm{EDC})$ and store at $-20{ }^{\circ} \mathrm{C}$. Thaw and combine $0.1 \mathrm{~g}$ EDC with $2.6 \mathrm{ml}$ of the MES-NHS solution just before use (make fresh solution for each use).

7. Extracellular matrix coating solution: Prepare a stock solution of $10 \mathrm{mg} / \mathrm{ml}$ fibronectin (F114l-1MG Sigma) in $\mathrm{ddH}_{2} \mathrm{O}$ and make $100 \mu \mathrm{l}$ aliquots for $-20{ }^{\circ} \mathrm{C}$ storage (see Note 3 ).

8. Quenching solution: ethanolamine $0.32 \%$ diluted in $1 \times \mathrm{PBS}$.

9. Neural crest culture medium: Danilchik's for Amy medium (DFA) $10 \times: 530 \mathrm{mM} \mathrm{NaCl}, 50 \mathrm{mM} \mathrm{Na}_{2} \mathrm{CO}_{3}, 45 \mathrm{mM}$ potassium Gluconate, $320 \mathrm{mM}$ sodium Gluconate, $10 \mathrm{mM} \mathrm{MgSO}$, $10 \mathrm{mM} \mathrm{CaCl}_{2}, 1 \% \mathrm{BSA}$; adjusted to $\mathrm{pH} 8.3$ with $1 \mathrm{M}$ Bicine. We recommend preparing $500 \mathrm{ml}$ of DFA $10 \times$ and storage in $45 \mathrm{ml}$ at $-20{ }^{\circ} \mathrm{C}$. In order to prepare DFA $\mathrm{l} \times$, after thawing, add $1000 \mathrm{U}$ penicillin and $100 \mu \mathrm{g} / \mathrm{ml}$ streptomycin, dilute in distilled water and pass it to through a $0.2 \mu \mathrm{m}$ filter. Prepare $50 \mathrm{ml}$ aliquots and store them at $-20{ }^{\circ} \mathrm{C}$.

2.4 Neural Crest Dissection Tools and Protocol
The tools and protocol for Xenopus cranial neural crest cells dissection were previously described in Barriga et al. [16]. 


\subsection{Image and Statistical Analysis}

1. ImageJ or Fiji.

2. Statistical software (e.g., Microsoft Excel, GraphPad Prism, SPSS, Numbers).

\section{Methods}

\subsection{Mesoderm Targeted Injections and Validation}

3.1.1 Preparation for Microinjection

\subsubsection{Setting Up the Microinjector}

\subsubsection{Microinjection}

1. Perform in vitro fertilization (IVF) of $X$. laevis eggs and de-jelly the embryos as previously described [17].

2. After de-jellying the embryos, transfer them into a petri dish containing embryo injection media and keep them at $14{ }^{\circ} \mathrm{C}$ while preparing the microinjector and filling up the microinjection needles ( see Note 4 ).

3 . While the embryos are developing, prepare a solution containing a mixture of $0.1 \mathrm{ng} / \mathrm{nl}$ of Dsh-DEP ${ }^{+}$and $0.025 \mathrm{ng} / \mathrm{nl}$ of membrane GFP (mGFP) mRNAs in RNAse-free water. Prepare another solution containing mGFP mRNA $(0.025 \mathrm{ng} / \mathrm{nl})$ only to inject as a control (when needed). Keep solutions on ice until their use for injection.

1. Switch on the microinjector.

2. Set the microinjector for $0.2 \mathrm{~s}$ per pulse and then press balance.

3. Calibrate the needle before microinjection, as previously described [18]. This is an important step to ensure that the same volume is delivered in each microinjection ( see Note 5).

4. Fill the needle with the required volume to inject.

1. Place embryos from step 2 (Subheading 3.1.1) under the stereoscope that will be used for microinjection. Use forceps to orient the embryos with the animal side up. The dorsal side of the embryo can be distinguished from the ventral side by the presence of lighter pigmentation [19].

2. To inhibit disheveled specifically in the mesoderm and label the cells in this region for in vivo analyses, microinjection needs to be performed at 16-cell (stage 5 ) in the two dorso-vegetal blastomeres (V2.1 and V2.2, according to previously described fate maps $[20,21]$ ) with $5-10 \mathrm{nl}$ of control or Dsh-DEP ${ }^{+}$at the concentrations mentioned in step 3 of Subheading 3.1.1 (Fig. la) (see Note 6).

3. Injected embryos are incubated for at least $3 \mathrm{~h}$ at $14{ }^{\circ} \mathrm{C}$ in injection media ( see Note 7).

4. Replace the injection medium with embryo growth medium and keep at $14^{\circ} \mathrm{C}$ until reaching migratory stages (i.e., stages 21 or 22). Embryos can be staged according to Nieuwkoop and Faber [22]. 


\section{a}
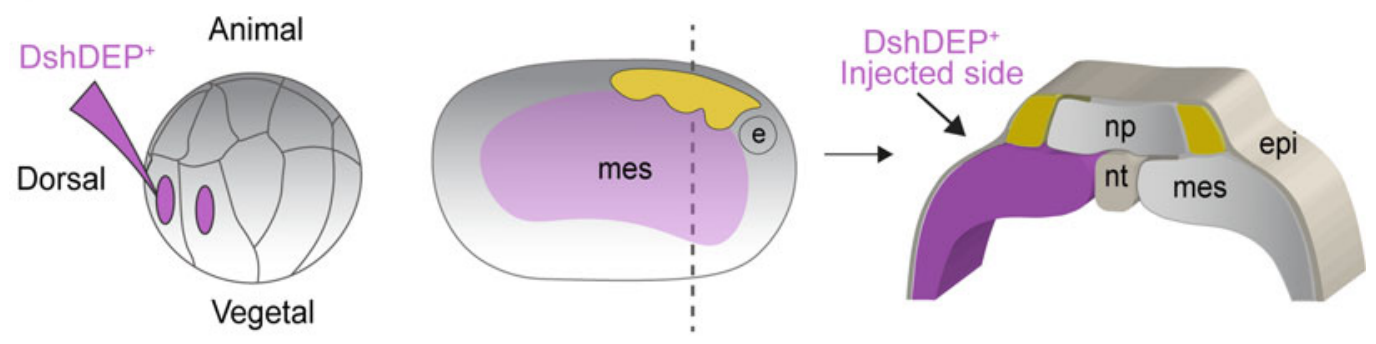

b

\section{Fibronectin/NC/mGFP}

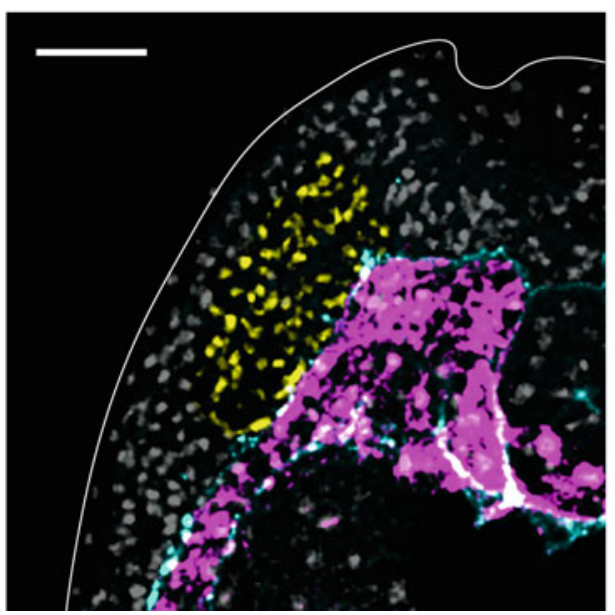

Fibronectin/mGFP

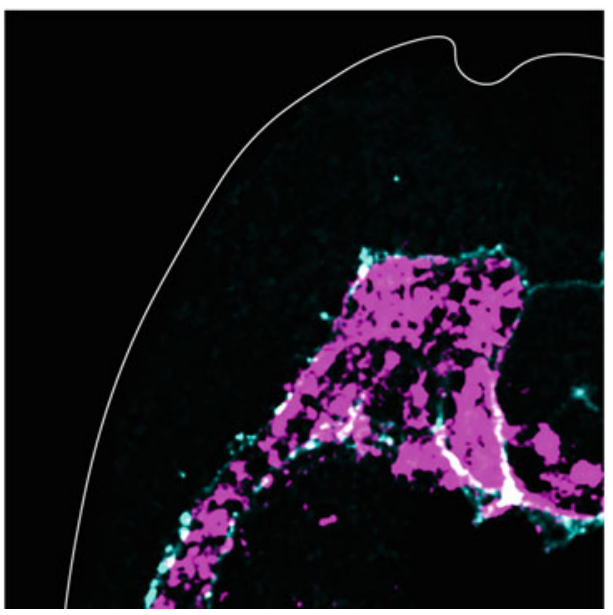

Fig. 1 Procedure and expected results for mesoderm targeted injections. (a) Schematic representation of a blastula embryo showing targeted injections and the resulting labeling at late neurula embryo. Magenta highlights the blastomeres required to be injected in order to target the anterior mesoderm in one side of the embryo. Dashed line indicates the plane of transversal section, which is shown on the right. $n c$ neural crest, $n p$ neural plate, epi epidermis, mes mesoderm, $n t$ notochord, $e$ eye (b) Confocal images of a transversal cryosection across stage 20 embryos showing membrane GFP localization in the mesoderm (pseudo-colored in magenta). Neural crest cells are pseudo-stained in yellow and fibronectin staining is shown in cyan. A white line is used to highlight the border of each section. Scale bar: $100 \mu \mathrm{m}$

3.1.4 Confirmation of Injection Accuracy by Immunofluorescent Staining of Fibronectin
One way to assess the effective delivery of targeted injections is to perform an immunostaining against fibronectin with nuclear labeling with DAPI for both control and Dsh-DEP ${ }^{+}$injected embryos at migratory stages. Fibronectin surrounds the neural crest, mesoderm, notochord and other tissues at these stages; hence it is an excellent marker to highlight our regions of interest (ROIs).

1. Fix the embryos for $2 \mathrm{~h}$ at room temperature or overnight at $4{ }^{\circ} \mathrm{C}$ (in a solution containing $4 \%$ formaldehyde in PBS- $0.3 \%$ Triton X 100) (see Note 8 ). 
3.2 Analyze

the Impact of PCP

Inhibition

on Mesoderm Cell

Density In Vivo
2. Process the embryos for cryosectioning as described in [6]; 30to 40 -micron thick sections are recommended for this particular experiment.

3. Allow the sections to dry overnight at room temperature.

4. Remove gelatin by incubating the slides at $37{ }^{\circ} \mathrm{C}$ in PBS (no detergent-see Note 9) two times for $25 \mathrm{~min}$ each time. Immediately place the sections in a slide mailer containing PBS $1 \times$ (always avoid them drying).

5. Block the sections for $30 \mathrm{~min}$ with $120 \mu \mathrm{l}$ of $10 \%$ normal goat serum (NGS) and cover the samples with parafilm (see Note 10). Perform the incubation in a humid chamber (see Note 11).

6. Remove the parafilm and exchange the blocking reagent for $150 \mu \mathrm{l}$ of the primary antibody solution and incubate overnight at $4{ }^{\circ} \mathrm{C}$ in the humid chamber and covered with parafilm.

7. Wash the primary antibody 4 times with $5 \mathrm{~min}$ each with PBS-0.1\% Tween 20 (see Note 12).

8. Incubate for $2 \mathrm{~h}$ at room temperature with the secondary antibody solution with DAPI (see Note 13).

9. Wash away the excess of secondary antibody as above and mount the samples in mounting medium (i.e., Mowiol, Vectashiled, etc.).

10. Image the stained sections with a fluorescence microscope (confocal is recommended). An example of the expected results is shown in Fig. $1 b$.

Previous work showed that Dsh-DEP ${ }^{+}$inhibits PCP signaling [9]. Hence by targeted injections of Dsh-DEP ${ }^{+}$into the mesoderm we expect to alter cell polarity of the mesodermal cells. Our work has previously demonstrated that one of the impacts of PCP inhibition in the mesoderm is to decrease mesodermal cell density in the anterior region of the embryonic midline which correlates with low stiffness values in this tissue [6]. Thus, we describe how to determine the impact of PCP inhibition on mesoderm cell density in this section and stiffness in the next section.

1. Process control or Dsh-DEP ${ }^{+}$injected embryos for cryosectioning and fibronectin immunofluorescence as described above.

2. Acquire Z-stack confocal images of the dorsolateral region of each section. The area for imaging is as shown in Figs. 1 and 2.

3. Open the images in ImageJ, generate a $\mathrm{z}$-projection (sum is recommended) by going to Image $>$ Stacks $>$ Z Project $>$ Sum Slices. Select the start and stop slices. 

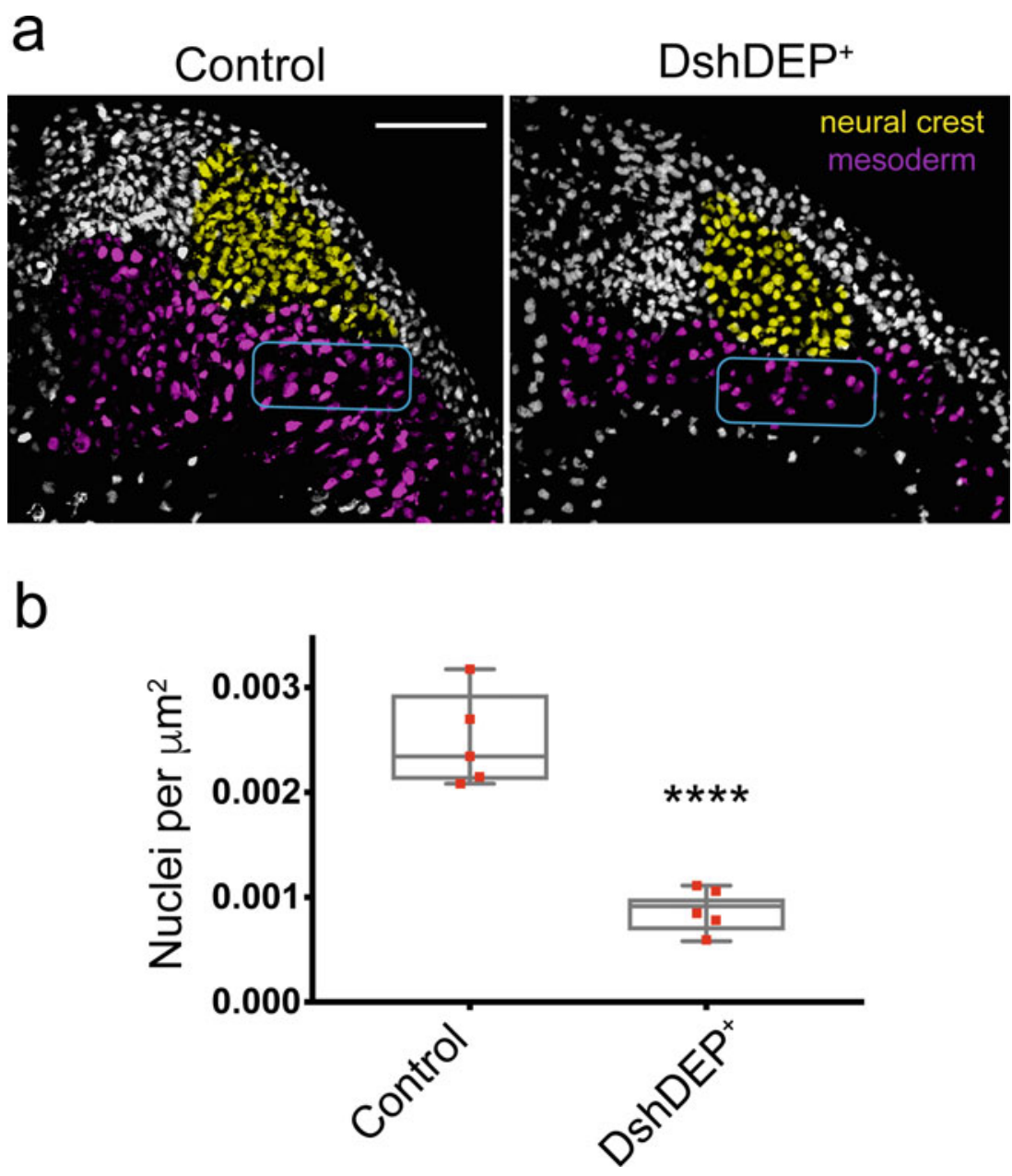

Fig. 2 Mesoderm cell density measurement and analyses of Dsh-DEP ${ }^{+}$injected embryos. (a) Confocal images of a transversal cryosection across Control and Dsh-DEP ${ }^{+}$injected embryos. Images display pseudo-stained nuclei for neural crest (yellow) and mesoderm (magenta). Regions inside the blue rectangle demarcates an example of the Rols selected for cell density quantifications. (b) Boxplot of mesoderm cell density measurements for control and Dsh-DEP ${ }^{+}$conditions (unpaired $t$ test, ${ }^{\star \star \star \star} P<0.0001$ ). Scale bar: $100 \mu \mathrm{m}$

4. Select a region of interest (ROI) in the mesoderm under the neural crest (cyan box in Fig. 2a). Use the same ROI size for all the conditions.

5. Calculate nuclei density in the selected ROI by counting the total number of nuclei within this region and dividing this number by the area of the selected ROI. Cell density can be then expressed in nuclei per $\mu \mathrm{m}^{2}$.

6. Finally plot the mean nuclei per $\mu \mathrm{m}^{2}$ of each condition, as shown in Fig. 2b, by using a statistical software (e.g., GraphPad, excel, etc.). 


\subsection{Measuring the Impact of PCP Inhibition \\ on Mesoderm Stiffening by In Vivo Atomic Force Microscopy (iAFM)}

\subsubsection{Initializing the Nanosurf Flex-ANA System (See Note 14)}

\subsubsection{Calibrating} the Cantilever in the Nanosurf Flex-ANA System
Atomic force microscopy involves scanning a surface using a probe to determine the mechanical properties of materials [15]. Owing to the wide range of AFM devices available, most of the published AFM protocols skip the measuring and machine handling procedures. This makes sense as these steps are highly variable from system to system. Regardless, in this protocol we provide the main steps and tips for using our device, the automated atomic force microscope Flex-ANA system by Nanosurf (ATS204 stage). The Flex-ANA system confers great advantages comparing with other systems, including an automated atomic force microscope coupled with a user-friendly software with a straight-forward workflow, easy cantilever calibration and real-time data analysis during the measurement. We will also focus on important aspects that have to be considered independently of the AFM system, including how to choose the type of the cantilever, how to prepare the samples (in this case, how to mount and hold an embryo), how to select the region of measurement, which acquisition parameters (e.g., the magnitude of force) should be used, and finally how to process and display the data.

1. Start the ANA software by pressing the ANA-icon. If a new experiment will be performed, the program will create a new folder. Otherwise, it is possible to open a pre-existing experiment and add new conditions.

2. Choose between Basic and Expert workflow (see Note 15).

3. Proceed through the steps indicated by the wizard (see Note 16). Although this wizard is very "user friendly," it might display small issues which can be easily addressed (see Notes 17-19).

1. Select the cantilever, the environment, and the temperature at which the system is operating ( see Note 20).

2. Align the laser to the cantilever (see Note 21) and center it on the photodetector by using the laser alignment tool. Take advantage of the top and lateral view cameras, which are included in the FlexANA device. Then proceed to the cantilever calibration every time you start a new session or if any issue arises while measuring a given data set.

3. Spring constant (k) $[\mathrm{N} / \mathrm{m}]$ calibration: cantilever spring constant (stiffness of the cantilever) has to be determined for conversion of metric cantilever deflection (deflection sensitivity) into units of force using the Sader method based on "Thermal Noise." While a reference value is provided, this value generally refers to a $(k)$ calculated in a dry/air interface. Since our measurements are performed in embryo media, it is highly recommended to estimate $(k)$ in this media by following 
the steps suggested by the wizard: first press "Start" in the "Thermal tuning" section in order to generate a thermal noise spectrum where the thermal fluctuations of the cantilever as a function of the frequency $(\mathrm{Hz})$ are plotted (see Note 22); then, the thermal resonance curve is fitted to a single harmonic oscillator model (Lorentz function), providing the resonance frequency of the cantilever and the quality (Q)-factor necessary to calculate the spring constant using the Sader Method [23]. Once the spring constant value is stabilized, press "Stop" and then press "Use results" to set the calculated spring constant.

4. Deflection sensitivity (ds) $[\mathrm{nm} / \mathrm{V}]$ calibration: the deflection sensitivity of the detectors has to be calibrated to know how many nanometers of motion correspond to a unit of voltage measured on the position sensitive photodetector (PSPD). For this, few steps are recommended: first, using the overview camera, acquire an image of the sample by pressing "Take Picture"; then, switch to experiment image viewer, select the position of the sample at which the sensitivity calibration will be performed to bring the cantilever over the sample (glass is highly recommended as its $(k)$ is normally assumed as "infinite"). Proceed to a "Deflection Cross-Talk Compensation" by pressing "Start" ( see Note 23). While in contact (see Note 24) and by using the "deflection calibration" wizard, start the deflection sensitivity calibration with recent settings by just pressing "Start deflection calibration"; otherwise, press "restore default parameters" or press "Next" and set the calibration parameters. The calculated sensitivity is displayed and the deviation between the defined force curves on a hard surface is given in \%. Once the process is concluded, press "Use Results." Based on pre-set thresholds the quality of the process will be assessed as "unusable," "low" or "high." We recommend using high quality deflection calibration results.

3.3.3 Set Up the Parameters and Perform a Test Measurement
Before measuring, it is always recommended to test the settings with a reference sample, e.g., glass. We use the Standard Spectroscopy Mode kit that contains three samples, a piece of glass (used for calibration) and two pieces of silicones made with pre-defined soft and stiff values (used to test the quality of each calibration). Here we describe the steps and parameters required to measure soft and stiff silicones, as these are the same steps that we will use in the embryo measurement section:

1. Place the Standard Spectroscopy Mode sample in the platform and using the top overview camera acquire an image of the sample (Fig. 3a) (see Note 25). Then place the AFM head back into position to align the sample with the cantilever. In the 
a

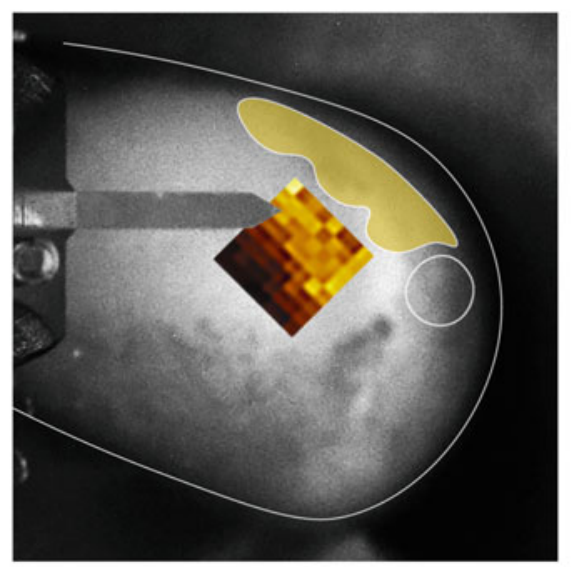

C

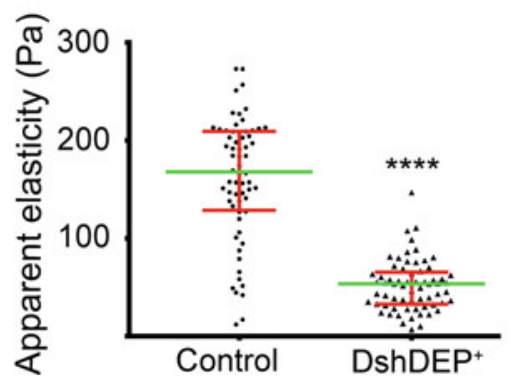

b
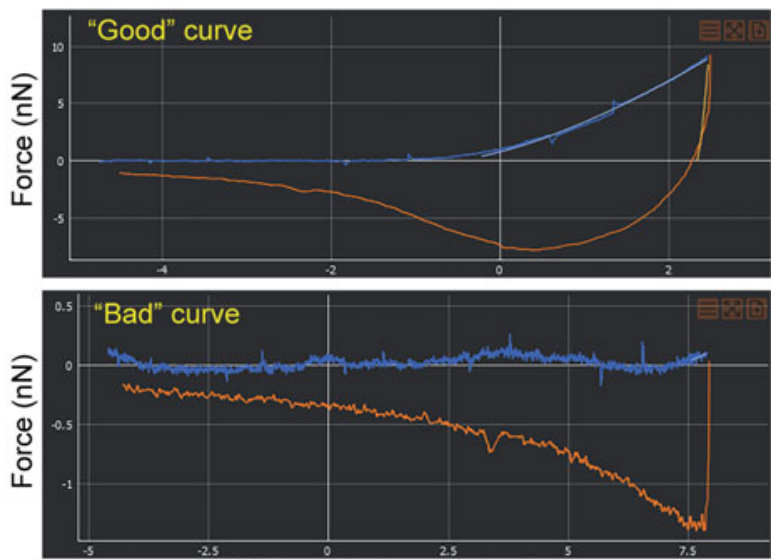

Tip Sample Separation $(\mu \mathrm{m})$

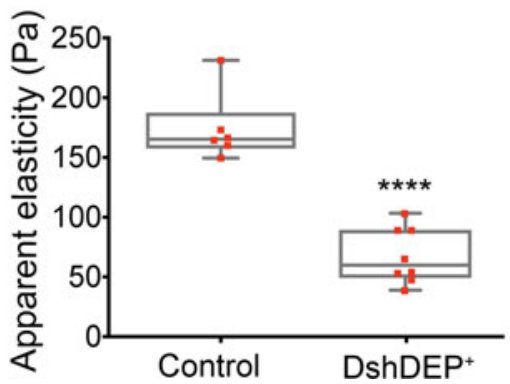

Fig. 3 AFM measurements in the mesoderm and analyses. (a) Picture of an embryo in which the skin has been removed to allow direct access of the mesoderm. The cantilever is shown in the image and a grid indicating the measured area (ROI) has been overlayed to the embryo. Notice that the heatmap shows that at migratory stages the mesoderm in front of neural crest cells is highly heterogeneous and it seems to become softer toward ventral regions (grid color-code: darker softer, brighter stiffer). The position of the neural crest is represented in yellow. (b) Representative examples of "good" and "bad" force ( $y$-axis) vs distance ( $x$-axis) curves. Blue indicates the approximation of the cantilever to the surface and red the retraction of the cantilever. (c) Results of AFM measurements are plotted in two ways to present the data as shown; on the left, a graph displaying the whole spread of data with the median highlighted in green and the interquartile range in red is shown (Mann-Whitney test, ${ }^{\star * \star \star} P<0.0001$ ); on the right, a boxplot where the averaged medians of the measurements acquired in each embryo has been plotted, little red squares represent the median of each embryo (unpaired $t$ test, ${ }^{\star \star \star \star} P<0.0001$ )

experiment planner window or image viewer, click into the image to select one or multiple ROIs ( see Note 26).

2. Before measuring, set the grid dimensions for each ROI. For Standard Spectroscopy Mode samples as well as for Xenopus embryos we recommend the following dimensions: Height: $50 \mu \mathrm{m}$; Width: $50 \mu \mathrm{m}$; Points per line:8; Number of lines: 8.

3. Acquisition parameters: we recommend using the following parameters: $10 \mathrm{nN}$ of maximum force; Ramp length: $10 \mu \mathrm{m}$ 
3.3.4 Determining Mesodermal Apparent Elasticity of Control and Dsh-DEP+ Embryos

\subsubsection{In Vivo Atomic} Force Microscopy (iAFM) Data Analysis and Presentation (forward) and $-10 \mu \mathrm{m}$ (backward); Ramp speed: $5 \mu \mathrm{m} / \mathrm{s}$ (forward) and $50 \mu \mathrm{m} / \mathrm{s}$ (backward) (see Note 27). Also, in the data analysis tab, enable the elasticity model (Hertz), set the tip radius of the cantilever and press "calculate." This is very important to monitor the quality of the results while they are being acquired and for further data analysis.

4. Although the FlexANA device has an automated "find surface" feature, make sure to manually approach the sample to the cantilever before starting the measurement (see Note 28) and press "Start measurement."

1. Place the control or Dsh-DEP ${ }^{+}$injected embryos in a dish with plasticine, containing MMR $0.1 \times$ and remove the epidermis around the vicinity of the neural crest as previously described $[6,16]$ and as shown in Fig. 3a (see Note 29).

2. Bring the cantilever to the region of the mesoderm that the neural crest will use as a substrate to migrate. The sample should look as in Fig. 3a.

\section{Apply the measurement as described in Subheading 3.3.3.}

AFM data analysis will depend on the devise and quality of results obtained by each user. Nonetheless we emphasize here the general aspects that might be considered as good practice in processing and storing bioAFM data. This is relevant, since most articles just provide a chart where values obtained from each curve are plotted. Since not all curves in a grid are always useful for analyses (Fig. 3b), we believe that it is important to provide raw data including all the curves obtained per embryo as well as those used to build the final chart where the filtered data are presented. This can help to understand the quality of the data and the reproducibility from embryo to embryo. It also facilitates data re-analysis by other authors. In addition, these guidelines can contribute to increasing reproducibility and transparency of AFM data. Here we provide the steps that we think will pave the path for good practices in bioAFM:

1. For every ROI/grid we aim to obtain 64 respective force distant curves and each grid is represented by heatmap. This $8 \times 8$ grid provides a spatial representation of the distribution of each one of the 64 data points acquired (Fig. 3a). Due to the distinct topology and adhesivity of the tissues, not all the measurements in the grid are suitable to be quantified. That is why prior analysis curves require to be sorted. There is a wide variety of curves shapes, however in Fig. $3 \mathrm{~b}$ we provide examples of what we call "good" and "bad" curves. Force spectroscopy curve analysis has been extensively discussed by others [24-26]. 


\subsection{Ex Vivo Analyses of NC Polarity Using Hydrogels}

3.4.1 Glass Coating

3.4.2 Polyacrylamide Hydrogels Preparation
2. At least three types of curves can emerge from the measurements: (1) "good curves," which are the ones with a good shape, indentation and low adhesion. We strongly recommend that you base your initial statistical analysis with the "good curves"; (2) the so-called bad curves which as stated are curves with bad shapes as a consequence of the inherent noises present in biological samples. We advise not using these curves; and (3) curves which are not "great" but can be improved with a bit of data analysis so that they can be used for final statistical processing. Further AFM data analysis has been extensively reviewed by other groups [24-26].

3. With "good curves," you can extract data to build a chart (e.g., in the FlexANA software this piece of data is named Forward E-Modul provided in $[\mathrm{Pa}]$ ). We recommend that each condition is repeated at least three times with at least three embryos for each independent experiment and three grids per embryo. Examples of data spread and box plot for data presentation are provided in Fig. 3c ( see Note 30).

4. Prepare two zip files containing the raw data (curves and their respective embryo image), one for the 64 measurements and the other for the selected curves. When publishing, make sure that both files are made available to the public.

1. In order to promote the attachment of gels to glass slides, coat $76 \mathrm{~mm} \times 24 \mathrm{~mm}$ glass slides with glass hydrophilic coating solution in the dark overnight at room temperature. The next day, wash the slides twice with $70 \%$ ethanol, air dry and store them at room temperature ( see Note 31);

2. Hydrophobic coating is required to prevent gels from sticking to the coverslips. For this, coat $13-\mathrm{mm}$ diameter $\times 0.1 \mathrm{~mm}$ glass coverslips with glass hydrophobic coating solution for 3-10 min at room temperature. Air dry (see Note 32);

1. Add $5 \mu$ of APS $10 \%$ to soft or stiff PAA mixes and gently mix the solutions, this will start the polymerization process (see Note 33);

2. Place a $13 \mu \mathrm{l}$ drop of each soft or stiff PAA mix in the hydrophilic glass slide and immediately cover it with a hydrophobic coverslip. Once polymerized (see Note 34), carefully remove the coverslip and add $10 \mathrm{mM}$ HEPES buffer to wash the excess of reagents after polymerization (see Note 35 ).

1. To covalently link fibronectin to the soft or stiff gels, remove HEPES buffer, cover the gels with gel functionalization solution, and put parafilm on top. Incubate the gels in a humidifier at room temperature for $40 \mathrm{~min}$ (see Note 36); 

3.4.4 Ex Vivo Analyses
of Neural Crest Polarity
2. Remove the parafilm and the medium and wash twice with PBS $1 \times$. Then replace the PBS with extracellular matrix coating solution and incubate in a humidifier at room temperature for l h 45 min (see Note 37);

3. Remove the extracellular matrix coating solution and replace it with quenching solution, cover the gels with parafilm and leave at room temperature for $15 \mathrm{~min}$. Wash twice with $10 \mathrm{mM}$ HEPES buffer with 5 min each. Remove the medium and place the gel in a culture dish containing neural crest culture medium and use soon ( see Note 38);

4. Dissect cephalic neural crests at pre-migratory stages as previously described in Barriga et al. [16]. Place these cells on either soft or stiff gels and wait 2-3 h for them to attach and spread in the gels.

A clear morphological parameter of cell polarity is the ability of the cells to break symmetry. Thus, we discuss below how to comparatively analyze cell roundness as a readout of their ability to polarize in soft and stiff hydrogels:

1. Open your image of interest in ImageJ/Fiji (Fig. 4a);

2. From the drawing tools, select * straight* line and draw a line across one cell nucleus. Ensure that the line spans the whole nucleus;

3. In the analyze tab, select "set scale." Select "known distance" to be the nominal length of the nucleus as "10"; then in the "unit of length" option, write "micron," since 10 micron is the typical nucleus diameter; select the box "Global"; and click "ok" (do not close ImageJ/Fiji). When "Global" is checked, the scale defined in this dialog is used for all images instead of just the active image;

4. Click on the figure outside your selection line to delete it and from the drawing tools, select *freehand* line and draw carefully along the contour of the cell;

5. In the analyze tab, select "set measurements" and select the box "shape descriptors." Then select "measure" (you can also click CONTROL $+\mathrm{M}$ ) and this will pop up a window with a list, where among other parameters the roundness and circularity index of cell are displayed.

6. Repeat this for all the cells that are being analyzed in each condition (soft and stiff in this case). Each time you select a cell press $(t)$ so that the morphological parameters of the selected cell will be added to the ROI Manager. Then select all your particles in the ROI manager and click on "measure" to add them into the list. 

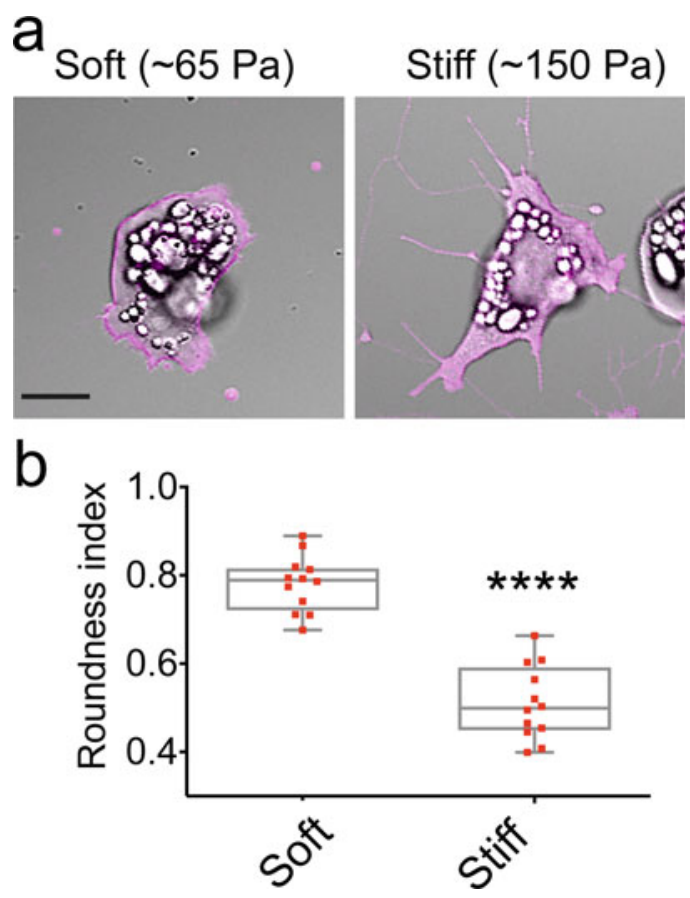

Fig. 4 Ex vivo analyses of neural crest polarity. (a) Confocal slices of labeled single neural crest cells plated on soft or stiff gels (as labeled). Scale bar: $10 \mu \mathrm{m}$. (b) Quantifications of single neural crest cells plated on soft or stiff gels. Boxplots show median values of the cell roundness in each condition represented by red squares (Mann-Whitney test, ${ }^{\star \star \star \star} P<0.0001$ )

7. From the generated list, plot the average circularity or roundness index values for each condition (soft/stiff), using a statistical software (e.g., Microsoft Excel, GraphPad Prism, SPSS, Numbers). A circularity index of 1 would mean that a cell is perfectly round, whereas a cell that is polarized will normally display values lower than 0.6 (Fig. 4b).

\section{Notes}

1. https://www.nanoandmore.com/Colloidal-NanomechanicsAFM-Probes.

This is a very user-friendly web page where cantilevers with spherical tips can be purchased from available selections or custom designs.

2. This solution requires to be freshly prepared since the NHS is extremely sensitive. Once prepared, you can immediately freeze and store at $-20{ }^{\circ} \mathrm{C}$ for up to 2 months.

3. Fibronectin solutions can be stored for at least a year at $2-8{ }^{\circ} \mathrm{C}$ if kept sterile. 
4. The temperature will influence the rate of development. This will allow you some time/flexibility as you can speed embryos up or down according to your needs. Optimal temperatures can be estimated using time/temperature charts (www. xenbase.org).

5 . It is good to maintain a pool of already calibrated needles (3-5 of them).

6. Injection needs to be done before the embryos reach 32-cells stage, as after that it becomes difficult to fate map each blastomere. While Ficoll is an ideal embryo media, an alternative is to just use $0.3 \times \mathrm{MMR}$, in case Ficoll is not available. However, for this particular experiment, Ficoll is highly recommended. Ficoll in the embryo injection media prevents leakage, as it collapses the vitelline space so that the embryo is not under pressure [19].

7. Overnight incubations with Ficoll are also acceptable but never let the embryos to gastrulate in this solution. While sometimes they would survive, at least in $70 \%$ of the cases this would lead to lethality.

8. Other fixatives such as trichloroacetic acid (TCA), paraformaldehyde (PFA), or glutaraldehyde may also work for anti-Fn.

9. Using detergent, i.e., Tween-20 or Triton-x 100 , to remove the excess of gelatin from the sections can cause the removal of the tissue itself, so avoiding the use of detergent is recommended.

10. This will minimize the volume of blocking reagent and antibody required in each step.

11. This chamber can be a pipette tip box with wet paper towels at the bottom and two pieces of Pasteur pipettes that serve as slide holders.

12. Rinses between washes are always recommended.

13. $1 \mu \mathrm{l}$ of anti-Mouse Alexa Fluor 555 and $1 \mu$ of DAPI every $350 \mu$ of $10 \%$ NGS

14. Removing and placing back the AFM head can cause the formation of bubbles when working in liquid interfaces. In addition, cantilever/laser dealignments can occur. Hence, be extremely careful when moving the head and checking the cantilever/laser alignment and the presence of bubbles between steps is highly recommended.

15. While the "Basic mode" strictly guides the user through the necessary steps to perform the measurements, the "Expert mode" allows more flexibility in controlling the cantilever calibration workflow and the parameters defined for force mapping. 
16. Wizard is very intuitive in this system that was indeed developed for non-expert users.

17. Select "Calibrate Stage/Camera" and proceed to stage referencing by pressing "Search Reference." This step is mandatory every time the system or the software is started. Before starting this procedure, make sure that the scan head is removed from the stage to prevent a crash of the cantilever into the sample.

18. When the stage reference search is successfully completed, proceed to "Camera lens correction." Select your objective from the list and use the checkerboard sample delivered along with the system. The default lens delivered with the system is pre-defined and the default correction profile is well suited for the default lens. If your objective is not in the list, use "New" to add a new one. In this case, press "New" to run the process of determining the lens distortion.

19. Perform the "Lens and Stage Correlation." Select the lens which should be correlated to the stage and use the high contrast sample delivered along with the system. Start the automatic correlation procedure. Once the correlation was successfully performed upon first installation and for the objective used, this step can be skipped. Repeat the procedure when changing the objective or if the optical components have been changed, moved, or replaced.

20. When doing measurements in liquid, perform a sensitive calibration for liquid.

21. When starting, the laser will not be necessarily in the position of the cantilever, meaning that the position of the laser cannot be seen. If this is the case, the first step of the laser alignment is to position the laser over the cantilever. For further details on how to proceed, visit https://www.nanosurf.com/en/video/ laser-and-detector-alignment. Once the laser is on the cantilever, the frontal or lateral cameras can be used to position the laser over the tip of the cantilever. Finding the laser can be challenging in a stand-alone version. Coupling to an inverted microscope helps both to find the laser and align it with the cantilever and detector.

22. It is important that the cantilever is retracted from the surface.

23. This step is relevant as it will determine what portion of the deflection observed after each $\mathrm{z}$ displacement of the cantilever corresponds to resistance opposed by the media. Hence, in our context we always make sure that we perform this step while the cantilever is in the liquid but not in contact with the surface.

24. Make sure you are in contact with the sample. 
25. A new experiment has to be created and assigned to a folder before taking the picture of a new sample. So, the picture and all the data/curves as well as logs of that particular experiment/sample will be saved in the same folder.

26. This device and software allow to acquire multiple ROIs with different grid parameters. One important observation is that the roughness of the region in between ROIs cannot be higher than $130 \mu \mathrm{m}$, since the max withdrawn distance is $150 \mu \mathrm{m}$. This will avoid lateral crashes of the cantilever with the sample when moving from one ROI to the other.

27. Since Xenopus tissue is highly adhesive, we recommend that the backward speed is performed with a higher value.

28. By doing this you will save time as normally the cantilever will be retracted to a maximum in between measurements (for security reasons that aim to avoid cantilever crashes).

29. The tissue needs to be as flat as possible in the ROIs.

30. Stiffness can be defined as the resistance of a material to deformation. Nonetheless, here we refer to the mechanical cues of the environment as "substrate apparent stiffness" and express it in $\mathrm{Pa}$ (because in native tissues we are at least measuring a convolution of elasticity and tension).

31. Coated glass slides can be used for up to 2 years. Nonetheless, for soft gels we recommend a maximum of l-week storage.

32. Coated coverslips should be used immediately, especially for soft gels.

33. Do not use APS after 1 week of storage, as the activity is reduced after that period and it will not be optimal for "soft" gels.

34. Polymerization time can vary. While $30-40$ min of polymerization is advised, we recommend having a sentinel gel to help monitor polymerization. We normally remove the glass from this gel after $30 \mathrm{~min}$ of polymerization, and if the polymerization is still not well achieved, we allow the other gels to polymerize for another $10 \mathrm{~min}$.

35. Never allow gels to completely dry, as this would drastically affect gel stiffness.

36. Every $10 \mathrm{~min}$, monitor the formation of air bubbles as they can generate unfunctionalized gel regions. To remove them, lift and drop the parafilm.

37. We recommend an incubation time of $1 \mathrm{~h} 45 \mathrm{~min}$, but a minimum of $1 \mathrm{~h} 30 \mathrm{~min}$ and a maximum of $2 \mathrm{~h}$ can be used.

38. While we recommend using immediately, gels can be store at $4{ }^{\circ} \mathrm{C}$ for up to 1 day (based on our tests, nonetheless other authors claim that they can be stored for longer). 
Acknowledgments

We would like to thank the IGC imaging and aquatic animal facilities. EHB lab receives funding from the European Research Council (ERC) under the European Union's Horizon 2020 research and innovation programme (grant agreement no. 950254), "EMBO IG Project Number 4765" and "la Caixa Junior Leader Incoming (94978)."

\section{References}

1. Ozair MZ, Kintner C, Brivanlou AH (2013) Neural induction and early patterning in vertebrates. Wiley Interdiscip Rev Dev Biol 2: 479-498. https://doi.org/10.1002/wdev.90

2. Gilbert SF (2010) Developmental biology. Sinauer Associates

3. Spemann H, Mangold H (2003) Induction of embryonic primordia by implantation of organizers from a different species. 1923. Int J Dev Biol 45:13-38. https://doi.org/10.1387/ ijdb.11291841

4. Saha MS, Spann CL, Grainger RM (1989) Embryonic lens induction: more than meets the optic vesicle. Cell Differ Dev 28:153-171. https://doi.org/10.1016/0922-3371(89) 90001-4

5. Steventon B, Carmona-Fontaine C, Mayor R (2005) Genetic network during neural crest induction: from cell specification to cell survival. Semin Cell Dev Biol 16:647-654. https://doi.org/10.1016/j.semcdb.2005. 06.001

6. Barriga EH, Franze K, Charras G, Mayor R (2018) Tissue stiffening coordinates morphogenesis by triggering collective cell migration in vivo. Nature 554:523-527. https://doi. org/10.1038/nature25742

7. Goodwin K, Nelson CM (2021) Mechanics of development. Dev Cell 56:240-250. https:// doi.org/10.1016/j.devcel.2020.11.025

8. Campàs $\mathrm{O}$ (2016) A toolbox to explore the mechanics of living embryonic tissues. Semin Cell Dev Biol 55:119-130. https://doi.org/ 10.1016/j.semcdb.2016.03.011

9. Shindo A, Wallingford JB (2014) PCP and septins compartmentalize cortical actomyosin to direct collective cell movement. Science 343:649-652. https://doi.org/10.1126/sci ence. 1243126

10. Behrndt M, Salbreux G, Campinho P, Hauschild R, Oswald F, Roensch J, Grill SW, Heisenberg C-P (2012) Forces driving epithelial spreading in zebrafish gastrulation. Science
338:257-260. https://doi.org/10.1126/sci ence. 1224143

11. Nishimura T, Honda H, Takeichi M (2012) Planar cell polarity links axes of spatial dynamics in neural-tube closure. Cell 149: 1084-1097. https://doi.org/10.1016/j.cell. 2012.04.021

12. Bertet C, Sulak L, Lecuit T (2004) Myosindependent junction remodelling controls planar cell intercalation and axis elongation. Nature 429:667-671. https://doi.org/10. 1038 /nature 02590

13. Barriga EH, Mayor R (2019) Adjustable viscoelasticity allows for efficient collective cell migration. Semin Cell Dev Biol 93:55-68. https://doi.org/10.1016/j.semcdb.2018. 05.027

14. Sokol SY (1996) Analysis of Dishevelled signalling pathways during Xenopus development. Curr Biol 6:1456-1467. https://doi.org/10. 1016/S0960-9822(96)00750-6

15. Franze K (2011) Atomic force microscopy and its contribution to understanding the development of the nervous system. Curr Opin Genet Dev 21:530-537. https://doi.org/10.1016/j. gde.2011.07.001

16. Barriga EH, Shellard A, Mayor R (2019) In vivo and in vitro quantitative analysis of neural crest cell migration. Methods Mol Biol 1976: 135-152. https://doi.org/10.1007/978-14939-9412-0_11

17. Wlizla M, McNamara S, Horb ME (2018) Generation and care of Xenopus laevis and Xenopus tropicalis embryos. In: Vleminckx K (ed) Xenopus. Springer, New York, NY, pp 19-32

18. Sive HL, Grainger RM, Harland RM (2010) Calibration of the injection volume for microinjection of Xenopus oocytes and embryos. Cold Spring Harb Protoc 2010:pdb. prot5537-pdb.prot5537. https://doi.org/10. $1101 /$ pdb.prot5537

19. Sive HL, Grainger RM, Harland RM (2010) Microinjection of Xenopus embryos. Cold 
Spring Harb Protoc 2010:pdb.ip81-pdb.ip81. https://doi.org/10.1101/pdb.ip81

20. Moody S (1987) Fates of the blastomeres of the 16-cell stage Xenopus embryo. Dev Biol 119:560-578

21. Bauer DV, Huang S, Moody SA (1994) The cleavage stage origin of Spemann's Organizer: analysis of the movements of blastomere clones before and during gastrulation in Xenopus. Development 120(5):1179-1189

22. Nieuwkoop PD, Faber J (1994) Normal table of Xenopus laevis (Daudin): a systematical and chronological survey of the development from the fertilized egg till the end of metamorphosis. Garland Pub, New York

23. Hutter JL, Bechhoefer J (1998) Calibration of atomic-force microscope tips. Rev Sci Instrum
64:1868. https://doi.org/10.1063/1. 1143970

24. Benítez R, Bolós VJ, Toca-Herrera J-L (2017) afmToolkit: an R package for automated AFM force-distance curves analysis. R J 9:291. https://doi.org/10.32614/RJ-2017-045

25. BenÍtez R, Moreno-flores S, BolÓs VJ, TocaHerrera JL (2013) A new automatic contact point detection algorithm for AFM force curves. Microsc Res Tech 76:870-876. https://doi.org/10.1002/jemt.22241

26. Kasas S, Riederer BM, Catsicas S, Cappella B, Dietler G (2000) Fuzzy logic algorithm to extract specific interaction forces from atomic force microscopy data. Rev Sci Instrum 71: 2082-2086. https://doi.org/10.1063/1. 1150583

Open Access This chapter is licensed under the terms of the Creative Commons Attribution 4.0 International License (http://creativecommons.org/licenses/by/4.0/), which permits use, sharing, adaptation, distribution and reproduction in any medium or format, as long as you give appropriate credit to the original author(s) and the source, provide a link to the Creative Commons licence and indicate if changes were made.

The images or other third party material in this chapter are included in the chapter's Creative Commons licence, unless indicated otherwise in a credit line to the material. If material is not included in the chapter's Creative Commons licence and your intended use is not permitted by statutory regulation or exceeds the permitted use, you will need to obtain permission directly from the copyright holder. 